\title{
Annals of Medicine
}

\section{Pre-pandemic mental and physical health as predictors of COVID-19 vaccine hesitancy: evidence from a UK-wide cohort study}

\section{G. David Batty, Ian J. Deary \& Drew Altschul}

To cite this article: G. David Batty, lan J. Deary \& Drew Altschul (2022) Pre-pandemic mental and physical health as predictors of COVID-19 vaccine hesitancy: evidence from a UK-wide cohort study, Annals of Medicine, 54:1, 274-282, DOI: 10.1080/07853890.2022.2027007

To link to this article: https://doi.org/10.1080/07853890.2022.2027007
(c) 2022 The Author(s). Published by Informa UK Limited, trading as Taylor \& Francis Group.

\section{曲 Published online: 24 Jan 2022.}

Submit your article to this journal

Џll Article views: 153

Q View related articles $\square$

View Crossmark data $\nearrow$ 


\title{
Pre-pandemic mental and physical health as predictors of COVID-19 vaccine hesitancy: evidence from a UK-wide cohort study
}

\author{
G. David Batty ${ }^{\mathrm{a}}$ (D) lan J. Deary ${ }^{\mathrm{b}}$ (D) and Drew Altschul ${ }^{\mathrm{C}}$
}

${ }^{a}$ Department of Epidemiology and Public Health, University College London, London, UK; ${ }^{b}$ Lothian Birth Cohorts, Department of Psychology, University of Edinburgh, Edinburgh, UK; 'Department of Psychology, University of Edinburgh, Edinburgh, UK

\begin{abstract}
Background: Although several predictors of COVID-19 vaccine hesitancy have been identified, the role of physical health and, particularly, mental health, is poorly understood.

Methods: We used individual-level data from a pandemic-focused investigation (COVID Survey), a prospective cohort study nested within the UK Understanding Society (Main Survey) project. In the week immediately following the announcement of successful testing of the first efficacious inoculation (Oxford University/AstraZeneca, November/December 2020), data on vaccine intentionality were collected in 12,035 individuals aged 16-95years. Pre-pandemic, study members had responded to enquiries about diagnoses of mental and physical health, including the completion of the 12-item General Health Questionnaire for symptoms of psychological distress (anxiety and depression). Peri-pandemic, individuals indicated whether they or someone in their household was shielding; that is, people judged by the UK National Health Service as being particularly clinically vulnerable who were therefore requested to remain at home. Intention to take up vaccination for COVID-19 was also self-reported.

Results: In an analytical sample of 11,955 people (6741 women), $15.4 \%$ indicated that they were vaccine-hesitant. Relative to their disease-free counterparts, shielding was associated with a $24 \%$ lower risk of being hesitant (odds ratio; $95 \%$ confidence interval: $0.76 ; 0.59,0.96$ ), after adjustment for a range of covariates which included age, education, and ethnicity. Corresponding results for cardiometabolic disease were $22 \%(0.78 ; 0.64,0.95)$, and for respiratory disease were $26 \%(0.74 ; 0.59,0.93)$. Having a pre-pandemic diagnosis of anxiety or depression, or a high score on the distress symptom scale, were all unrelated to the willingness to vaccinehesitancy.

Conclusions: People with a physical condition were more likely to take up the potential offer of a COVID-19 vaccination. These effects were not apparent for indices of mental health.

\section{KEY MESSAGES}

- In understanding predictors of COVID-19 vaccine hesitancy, the role of physical and mental health has not been well-examined despite both groups seemingly experiencing an elevated risk of the disease.

- In a large UK cohort study, people with a pre-pandemic physical condition were more likely to take up the theoretical offer of vaccination.

- There were no apparent effects for indices of pre-pandemic mental health.
\end{abstract}

\section{ARTICLE HISTORY}

Received 9 June 2021

Revised 27 December 2021

Accepted 4 January 2022

\section{KEYWORDS}

Mental health; physical health; COVID-19; vaccine hesitancy; cohort study

\section{Introduction}

Whereas it was established early in the COVID-19 pandemic that people with chronic physical illness experienced higher rates of hospitalisation for, and death from, the disease [1-4], more recent evidence suggests that the same may also be the case for people with mental health problems and those with a higher prevalence of psychological distress symptoms (anxiety and depression) [4-7]. There have therefore been calls to test the link between mental health and vaccine hesitancy [8], the concern being that any elevated burden of the disease in individuals with poor psychological health would be compounded if they were also reluctant to take up the vaccine.

CONTACT David Batty E.david.batty@ucl.ac.uk E Department of Epidemiology \& Public Health, University College London, 1-19 Torrington Place, London, WC1E 6BT, UK

(C) 2022 The Author(s). Published by Informa UK Limited, trading as Taylor \& Francis Group.

This is an Open Access article distributed under the terms of the Creative Commons Attribution-NonCommercial License (http://creativecommons.org/licenses/by-nc/4.0/), which permits unrestricted non-commercial use, distribution, and reproduction in any medium, provided the original work is properly cited. 
There are reasons to anticipate greater vaccine hesitancy in people with mental health problems. First, individuals with psychiatric morbidity and symptoms of distress generally tend to have a lower prevalence of health-protecting behaviours. Relative to their unaffected counterparts, for instance, they are more likely to smoke, take less exercise, have an imprudent diet, and be obese [9-11]. Second, people with mental health issues also appear to be less likely to take up the offer of health screening [12], although this is not a universal observation [13]. Lastly, of perhaps most relevance, in a study of influenza inoculation, users of an outpatient psychiatry clinic had markedly lower take-up than the general population [14].

Collectively, these observations provide a prima facie case that people with psychological health problems may be somewhat more hesitant when offered vaccination against COVID-19. Given a modest evidence base revealing inconsistent findings $[15,16]$, in a large, general-population-based UK sample we examined the relationships of mental health diagnosis and symptoms of mental distress with vaccine hesitancy. For the purposes of comparison, we also present the association between somatic illness and vaccine hesitancy; in the few relevant studies, somatic illness has been associated with lower levels of hesitancy $[17,18]$. Importantly, collection of data on vaccine intention in the present study took place following the announcement of successful testing of the Oxford University/ AstraZeneca vaccine, which was widely and prominently publicised. Therefore, the present survey concerning vaccination hesitancy was taken at a time when the future offer of vaccination was no longer merely hypothetical.

\section{Methods}

Understanding Society, also known as the UK Household Longitudinal Study, is a nationally representative, on-going, open, cohort study (hereinafter, the 'Main Survey'). Scientific leadership was provided by the Institute for Social and Economic Research, University of Essex, and data were collected by NatCen and Kantar Public [19]. The study was initiated in 2009 when adults aged 16 years or over in selected households were invited to participate. Study participants have been interviewed annually using different approaches (online, face-to-face or telephone survey) [19]. At Wave 1, face-to-face interviews were completed with 47750 individuals for an individual response within participating households of $80 \%$.
Households who had participated in at least one of the two most recent waves of data collection (wave 8 , 2016-18; wave 9, 2017-19) comprised the target sample for a pandemic-focused study initiated in April 2020 (hereinafter, the 'COVID Survey') [20,21]. The derivation of the present analytical sample from the Main and COVID Surveys is given in Figure 1. The University of Essex Ethics Committee gave approval for the COVID-orientated surveys (ETH1920-1271); no further ethical permissions were required for the present analyses of anonymised data. The return of a completed questionnaire was taken as implicit consent for participation in the COVID Surveys.

The COVID Surveys took place monthly/bimonthly between April (wave 1) and November 2020 (wave 6), with questions on vaccine intention first administered in the latest tranche of data collection when study members were aged 16-95 years (mean 53) [21]. Data collection in wave 6 (starting 24th November) commenced the day immediately following the announcement of the efficacy of the Oxford University/ AstraZeneca vaccine [22]. Data collection continued for one week, obtaining information from a total of 12,035 individuals of 19,294 invitations issued (response proportion 62\%) [21].

\section{Assessment of mental and physical morbidity}

Study members indicated if a physician or other health professional had ever informed them that they had a psychiatric problem, which included anxiety, depression, psychosis or schizophrenia, bipolar disorder or manic depression, an eating disorder, post-traumatic stress disorder, or any other mental illness (wave 10, 2019-20; Main Survey). With a low prevalence of hesitancy for selected conditions, we aggregated the latter five mental health groups. Self-reports of a physician diagnosis of mental illness, in particular depression, show reasonable agreement with a structured clinical interview $(61 \%$ sensitivity, $89.5 \%$ specificity, and a kappa statistic for overall agreement of 0.5) [23].

Psychological distress (wave 6, November 2020; COVID Survey) was ascertained using administration of the 12-item version of the General Health Questionnaire. Validated against standardised psychiatric interviews [24,25], this is a widely-used measure of psychological distress in population-based studies. Consistent with published analyses $[11,26,27]$, we used the following classifications: asymptomatic (score 0), sub-clinically symptomatic (score 1-3), symptomatic (score 4-6), and highly symptomatic (score 7-12). 


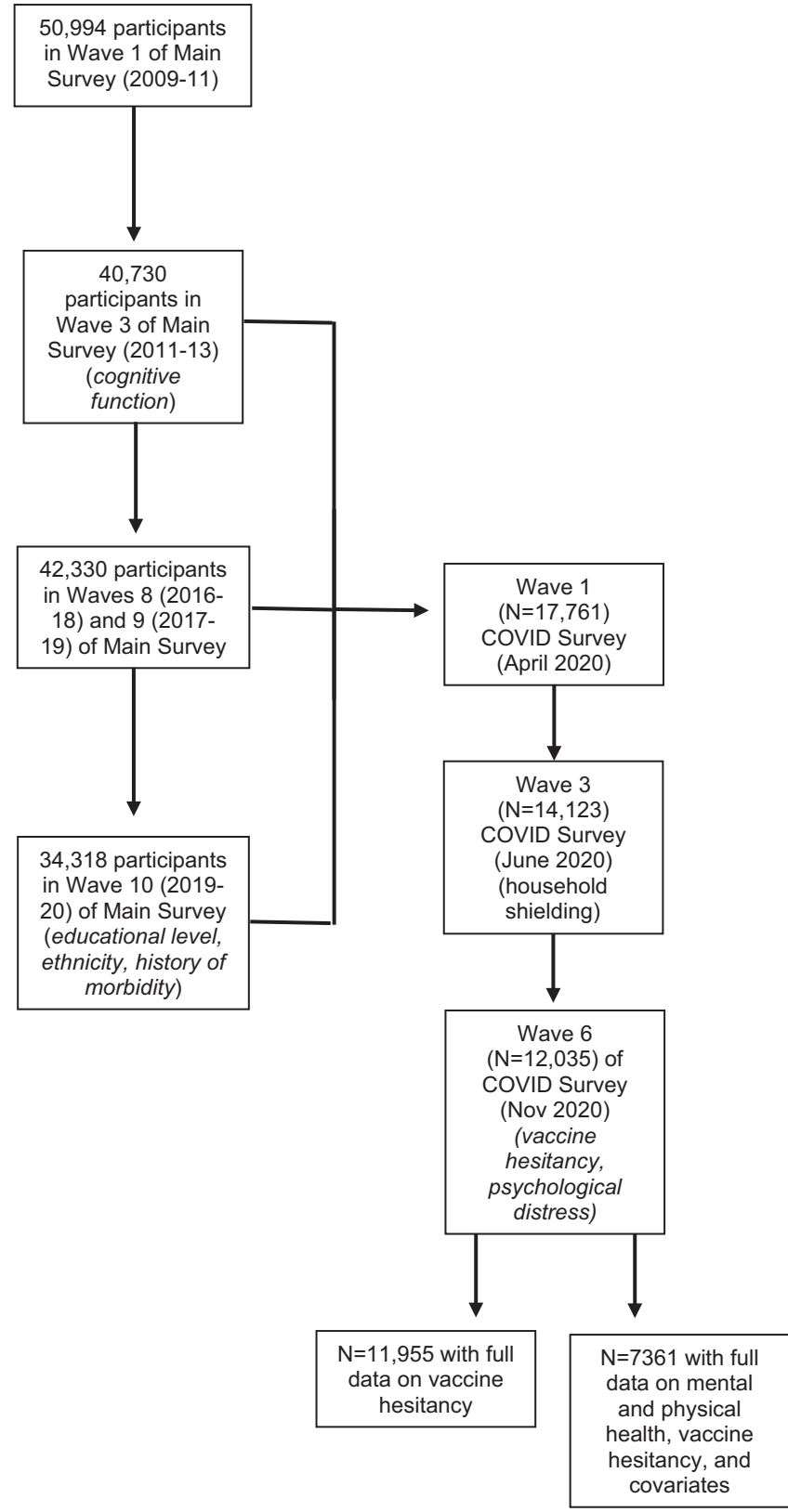

Figure 1. The flow of cohort members into the analytical sample: Main Survey and COVID Survey in Understanding Society.

A history of physical morbidity (wave 10, 2019-20; Main Survey) was based on self-report of physician diagnosis for a cardiometabolic condition (congestive heart failure, coronary heart disease, angina, heart attack or infarction, stroke, diabetes, and/or hypertension); respiratory disease (respiratory disease comprised bronchitis, emphysema, chronic obstructive pulmonary disease, and/or asthma); or cancer of any presentation. In other studies, these data reveal moderate to a high agreement with clinical records [28].

Lastly, based on their physical medical history, people judged as extremely clinically vulnerable to COVID19 were contacted by the UK National Health Service during the early stages of the pandemic and recommended to stay at home. Conditions that met the criteria for shielding included selected cancers, severe respiratory disorders such as cystic fibrosis, severe asthma, organ transplant recipients, and people with a disability such as Down's syndrome [29]. Study members were asked about the shielding status for themselves or a household member (waves 1-5, April to July 2020; COVID Surveys; denoted by yes/no).

\section{Assessment of covariates}

Covariates were self-reported and included age; sex (both wave 10, 2019-20; Main Survey); ethnicity (wave 10, Main Survey; denoted as white or non-white); and 
highest education level (wave 10, Main Survey; categorised as degree \& other higher degree, $A^{\prime}$ level or equivalent [Advanced Placement in the USA], GCSE or equivalent [Grade 10 in the USA], other qualification, and none). In the third wave of data collection in the Main Survey (2011-2013), six cognitive function tests were administered: immediate word recall and delayed word recall tasks; semantic verbal fluency; cognitive impairment; numerical reasoning skills; and fluid reasoning [30]. Representing a range of cognitive skills, these tests have been repeatedly deployed in largescale, population-based studies [31-35]. Using scores from the six tests, we generated a single general cognitive function variable $(g)$ for use in the present analyses [36].

\section{Assessment of vaccine hesitancy}

At wave 6 (November 2020) in the COVID Survey, study members were asked: "Imagine that a vaccine against COVID-19 was available for anyone who wanted it. How likely or unlikely would you be to take the vaccine?." Possible responses were "Very likely," "Likely," "Unlikely" and "Very unlikely." The latter two categories were combined to denote vaccine hesitancy.

\section{Statistical analyses}

To summarise the relation between mental morbidity, physical morbidity, and vaccine hesitancy, we used logistic regression to compute odds ratios with accompanying $95 \%$ confidence intervals. The most basic analyses were adjusted for age, sex, and ethnicity. Retaining these covariates, we then explored the impact of controlling separately and collectively for education, shielding status, and cognitive function. In analyses in which mental health was the exposure of interest, we adjusted for physical illness, and vice versa.

\section{Results}

In Table 1 we show study member characteristics according to vaccine intention in unadjusted analyses. In a sample of 11,955 individuals (6741 women) who responded in full to the enquiry regarding COVID-19 vaccine intentionality, $15.4 \%$ indicated that they were hesitant. Relative to the group who indicated a willingness to have the vaccine, those who were hesitant were more likely to be younger, female, from an ethnic minority background, be less well educated, and have a lower general cognitive function score. The hesitant were also less likely to have existing somatic morbidity, as indexed by cardiometabolic disease and cancer. Related, there was also a lower prevalence of shielding in the hesitant category (correlation between any physical morbidity and shielding in the present study: $\rho=0.12, p<.0001, N=10916)$. There was, however, little evidence of a difference in the prevalence of specific mental health diagnoses across the hesitant groups; only 'other' mental health conditions were more common in study members expressing hesitancy, but the absolute difference was marginal with statistical significance generated from the large numbers. People who declared themselves reticent in

Table 1. Study member characteristics according to COVID-19 vaccine hesitancy in Understanding Society.

\begin{tabular}{|c|c|c|c|}
\hline & \multicolumn{2}{|c|}{ Vaccine hesitant } & \multirow[b]{2}{*}{$P$ value } \\
\hline & Yes & No & \\
\hline Numbers of people & $1842(15.4)$ & $10113(84.6)$ & \\
\hline \multicolumn{4}{|l|}{ Demographic factors } \\
\hline Age, yr, mean (SD) & $45.0(14.5)$ & $54.6(15.6)$ & $<.0001$ \\
\hline Female & $1162(63.1)$ & $5530(54.7)$ & $<.0001$ \\
\hline Non-white ethnicity & $406(22.7)$ & $698(7.0)$ & $<.0001$ \\
\hline \multicolumn{4}{|l|}{ Socioeconomic factors } \\
\hline No higher education & $939(22.0)$ & $4298(6.9)$ & $<.0001$ \\
\hline \multicolumn{4}{|l|}{ Psychiatric morbidities } \\
\hline Anxiety & $85(4.0)$ & $404(4.6)$ & .153 \\
\hline Depression & $92(5.0)$ & $466(4.6)$ & .352 \\
\hline Other mental disorder & $36(1.9)$ & $121(1.2)$ & .007 \\
\hline Psychological distress symptoms, mean (SD) & $2.82(3.9)$ & $2.34(3.4)$ & $<.0001$ \\
\hline \multicolumn{4}{|l|}{ Physical morbidities } \\
\hline Cardiometabolic disease & $268(15.0)$ & $2513(25.2)$ & $<.0001$ \\
\hline Respiratory disease & $219(12.3)$ & $1372(13.8)$ & .144 \\
\hline Any cancer & $45(2.5)$ & $525(5.3)$ & $<.0001$ \\
\hline Shielding in the household & $196(10.6)$ & $1187(11.7)$ & $<.0001$ \\
\hline \multicolumn{4}{|l|}{ Cognitive function } \\
\hline$g$ factor, mean (SD) & $96.6(15.7)$ & $100.5(14.8)$ & $<.0001$ \\
\hline
\end{tabular}


Table 2. Odds ratios (95\% confidence intervals) for the relation of mental and physical health with later COVID19 vaccine hesitancy in Understanding Society $(N=7361)$.

\begin{tabular}{lccc}
\hline & Number hesitant/Total at risk & Age, sex, \& ethnicity & All covariates \\
\hline Psychiatric morbidity & & & \\
Anxiety & $50 / 324$ & $1.00(0.72,1.36)$ & $1.11(0.79,1.52)$ \\
Depression & $54 / 368$ & $0.99(0.72,1.33)$ & $1.12(0.81,1.53)$ \\
Other mental health condition(s) & $20 / 111$ & $1.08(0.64,1.75)$ & $1.21(0.71,1.97)$ \\
Any mental health condition & $71 / 491$ & $0.99(0.75,1.29)$ & $1.14(0.86,1.49)$ \\
Psychological distress & & $1.0($ ref $)$ & 1.0 (ref) \\
Asymptomatic (score 0) & $443 / 3339$ & $0.77(0.64,0.91)$ & $0.81(0.63,0.98)$ \\
Subclinically symptomatic (1-3) & $247 / 2256$ & $0.77(0.59,0.98)$ & $0.82(0.56,1.07)$ \\
Symptomatic (4-6) & $90 / 750$ & $1.05(0.85,1.28)$ & $1.12(0.92,1.33)$ \\
Highly symptomatic (7-12) & $173 / 1016$ & $<0.0001$ & 0.003 \\
P for quadratic association & & 0.251 & 0.075 \\
P for linear trend & & $0.93(0.81,1.06)$ & $0.88(0.75,1.02)$ \\
Per SD (3.5 points) decrease & $953 / 7361$ & & \\
Physical morbidity & & $0.82(0.67,0.99)$ & $0.78(0.64,0.95)$ \\
Cardiometabolic disease & $147 / 1905$ & $0.71(0.57,0.88)$ & $0.74(0.59,0.93)$ \\
Respiratory disease & $107 / 1034$ & $0.87(0.58,1.28)$ & $0.95(0.62,1.39)$ \\
Any cancer & $29 / 389$ & $0.72(0.61,0.85)$ & $0.72(0.60,0.85)$ \\
Any physical health condition & $225 / 2389$ & $0.81(0.63,1.03)$ & $0.76(0.59,0.96)$ \\
Shielding in household & $88 / 889$ &
\end{tabular}

All covariates are: age, sex, ethnicity, education, shielding status, and cognitive function. Effect estimates for physical morbidity and psychiatric morbidity were mutually-adjusted.

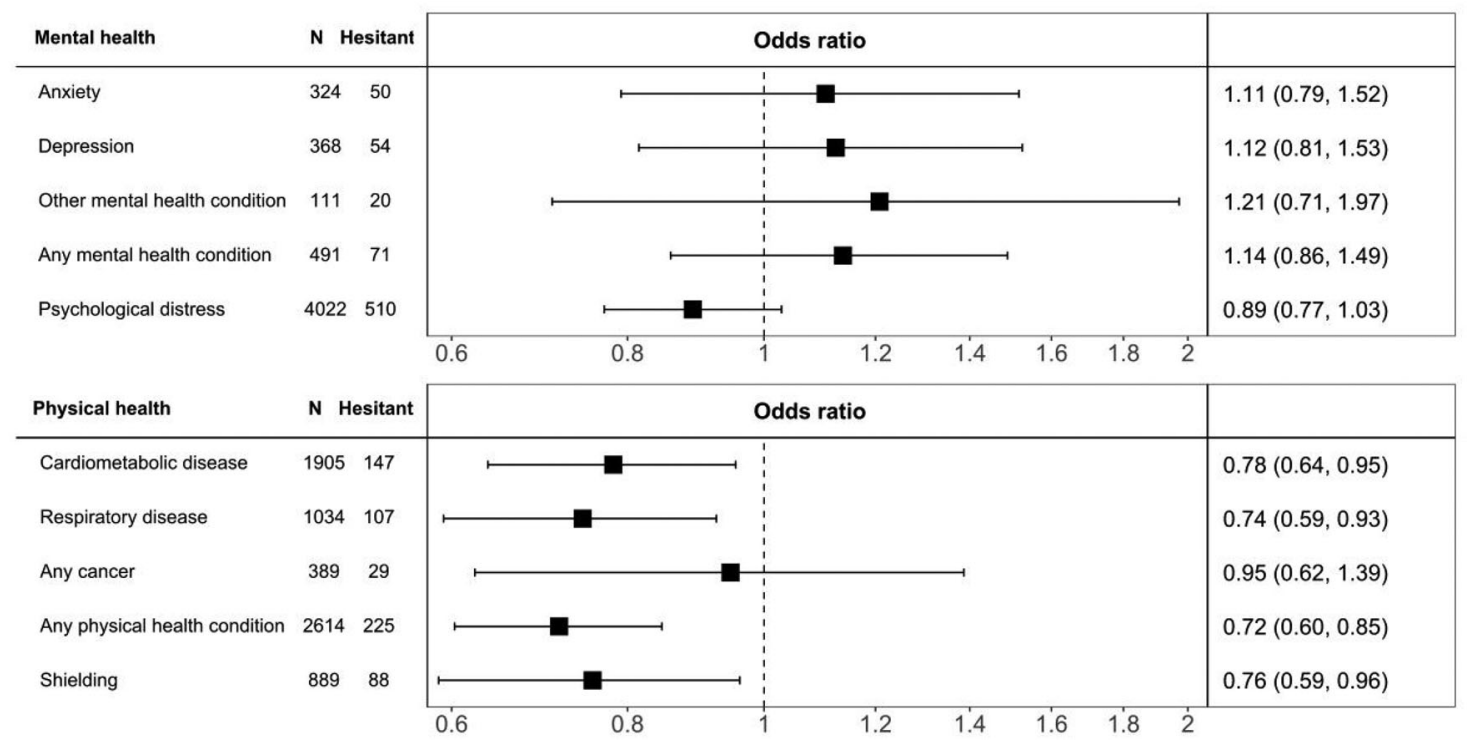

Figure 2. Multiply-adjusted odds ratios (95\% confidence intervals) for the relation of mental and physical health with later COVID-19 vaccine hesitancy in Understanding Society $(N=7361)$. The number of study members in this sample corresponds to those with complete data on all variables in the analyses. Effect estimates for physical morbidity and psychiatric morbidity were mutually-adjusted. For each morbidity, the referent group is those study members without the condition. An odds ratio below 1.0 indicates a factor was associated with a lower risk of vaccine hesitancy; a odds ratio greater than 1.0 indicates a factor was associated with a higher risk of hesitancy.

taking the vaccine when offered had slightly higher levels of psychological distress symptoms.

In Table 2 we used multiple regression analyses to explore the association between an existing diagnosis of morbidity and vaccine hesitancy. Relative to people without a physical condition, those with a diagnosis of cardiometabolic disease (odds ratio; 95\% confidence interval: $0.82 ; 0.67,0.99)$ or respiratory disease $(0.71$; $0.57,0.88$ ) were less like to have reported that they would decline an offer of vaccination, after adjustment for age, sex, and ethnicity. The associations of cancer and shielding with vaccine hesitancy were not statistically significant at conventional levels. Adjusting for a range of covariates (Table 2 and Figure 2) had little impact on these relationships; an exception was the regression coefficient for shielding becoming statistically significant such that people who were shielding were less vaccine-hesitant $(0.76 ; 0.59,0.96)$. The general lack of impact of controlling for individual covariates is shown in Table a1 (appendix). 


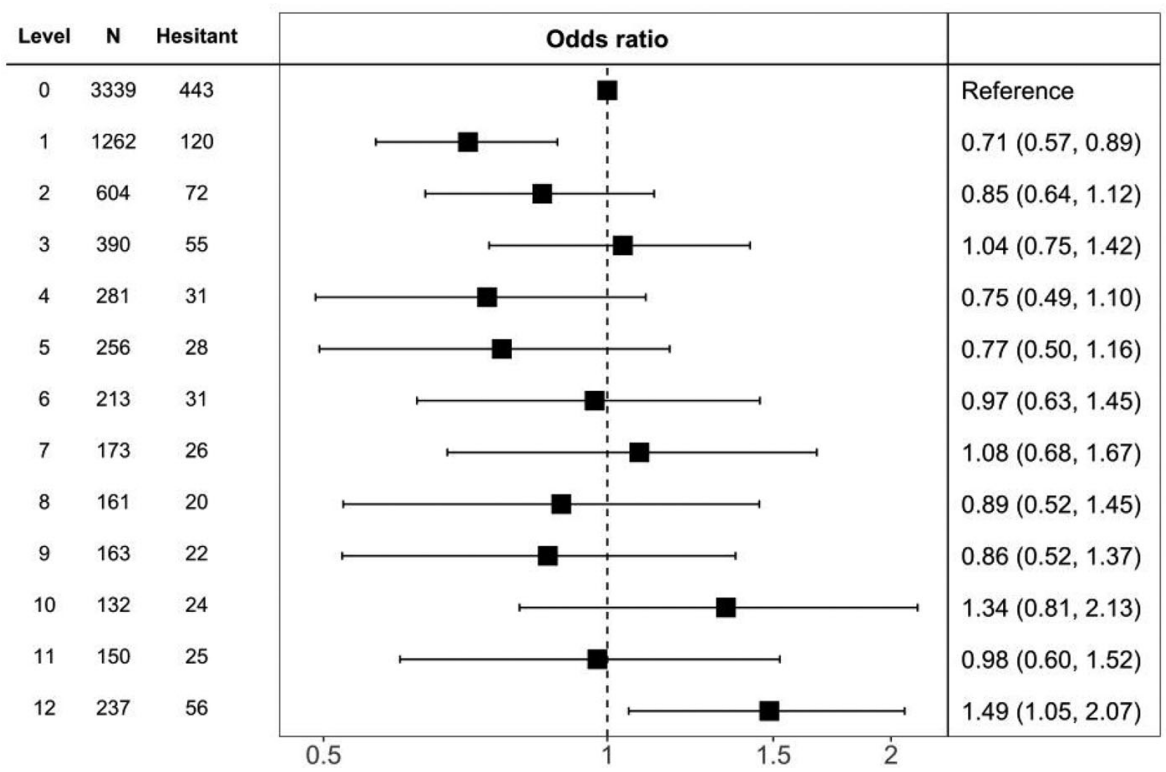

Figure 3. Multiply-adjusted odds ratios (95\% confidence intervals) for the relation of psychological distress with later COVID-19 vaccine hesitancy in Understanding Society $(N=7361)$. All covariates are: age, sex, ethnicity, education, somatic comorbidity, shielding, and cognitive function.

In analyses in which a diagnosis mental illness was the exposure of interest, none of the individual psychiatric conditions were related to vaccine hesitancy (Table 2 and Figure 2). Using the standard four-category schema for symptoms of psychological distress, however, there was some suggestion of a ' $U$ '-shaped effect, such that people who had either low or high scores on the distress scale were marginally more likely to be vaccine-hesitant, and those with moderate symptoms had the lowest likelihood ( $p$-value for quadratic relationship after multiple adjustments: 0.003). We further explored this association by using raw scores from the psychological distress scale (range 0-12). Based on this disaggregation, there was, however, no support for any relationship, linear or quadratic, between psychological distress and vaccine hesitancy (Figure 3).

\section{Discussion}

Our main finding was that, in data collected in the United Kingdom immediately following the announcement of the successful evaluation of the Oxford University/AstraZeneca vaccine, selected physical but not psychiatric morbidities were related to a lower likelihood of vaccine hesitancy. The results for mental health were unexpected, given that people with such morbidities are, as described, less likely to engage in health-protecting behaviours such as healthy lifestyle habits [11] and screening for the somatic disorder [12].

\section{Comparison with existing studies}

The notion that people with a long-standing physical condition are less likely to be vaccine-hesitant has been reported in other studies $[17,18]$. That we also recapitulated known associations with hesitancy such as being female [37-39], being younger [37,39], and from an ethnic minority group [21,39,40], gives us some confidence in our novel results for mental health. To the best of our knowledge, there have been two prior examinations of the relationship between mental health and vaccine hesitancy. Comprising two small cross-sectional studies from Ireland and the UK where data collection took place prior to the announcement of the successful testing of the first efficacious vaccination, study members were administered a very brief and unvalidated enquiry as to whether they had an experience of mental health problems. In that study, there was no clear evidence of a link [15]. In a Danish study in which vaccine takeup or the intention to do so in a group of individuals experiencing psychiatric care was compared with the general population, willingness was somewhat lower in the patient group [16]. Studies using data based on other vaccination programmes offer some insights into the present relationships. For instance, in a crosssectional study of patients with schizophrenia which took place during the $2009 \mathrm{H} 1 \mathrm{~N} 1$ influenza pandemic in Australia, three-quarters indicated that they were willing to be vaccinated [41]; however, in keeping 
with similar studies [42], the absence of a general population comparison group renders interpretation problematic. In a small cohort of socioeconomically disadvantaged mothers, those with mental health problems were seemingly less likely to have children with up-to-date vaccine coverage, although the association was weak and the study underpowered [43].

\section{Study strengths and weaknesses}

Whereas the present study has its strengths, including its size and the timing of data collection, there are also some weaknesses. First, we used vaccine intentionality as an indicator of vaccine uptake but the correlation between the two is imperfect. In a small-scale longitudinal study conducted during the period of the $2009 \mathrm{H} 1 \mathrm{~N} 1$ pandemic in Hong Kong, less than $10 \%$ of people who expressed a commitment to being inoculated reported that they had actually received a vaccination two months later [44]. Elsewhere, in a US adult population at high risk of seasonal influenza, around half of those intending to be vaccinated had received it within the following 5 months [45]. Second, there was inevitably some loss to follow-up (Figure 1). Whereas this attrition might have impacted upon the estimation of the prevalence of vaccine hesitancy, which is likely to be lower in our select sample relative to the general population [46], it is unlikely to have influenced our estimation of its relationship with mental and physical health. Thus, in other contexts, we have shown that highly-selected cohorts reveal very similar risk factor-outcome associations to those seen in studies with conventionally high responses [47]

In conclusion, we found that some somatic conditions but not mental health problems were related to a lower likelihood of being vaccine-hesitant against COVID-19.

\section{Acknowledgements}

Understanding Society is an initiative funded by the Economic and Social Research Council and various Government Departments, with scientific leadership provided by the Institute for Social and Economic Research, University of Essex, and survey delivery by NatCen Social Research and Kantar Public.

\section{Disclosure statement}

No potential conflict of interest was reported by the author(s).

\section{Author contributions}

GDB generated the idea for the present manuscript. DA built the dataset, conducted all analyses, and prepared the displayable items. All authors developed the analytical plan, and DA and IJD commented on a manuscript drafted by GDB.

\section{Funding}

GDB is supported by the UK Medical Research Council (MR/ P023444/1) and the US National Institute on Ageing (1R56AG052519-01; 1R01AG052519-01A1); and IJD by the UK Medical Research Council (MR/R024065/1), UK Economic and Social Research Council (ES/S015604/1), and the US National Institute on Ageing (1R01AG054628-01A1). These funders, who provided no direct financial or material support for the work, had no role in study design, data collection, data analysis, data interpretation, or report preparation.

\section{ORCID}

G. David Batty (D) http://orcid.org/0000-0003-1822-5753 lan J. Deary (iD http://orcid.org/0000-0002-1733-263X

Drew Altschul iD http://orcid.org/0000-0001-7053-4209

\section{Data availability statement}

The data that support the findings of this study are openly available from the UK Data Service (https://www.ukdataservice.ac.uk/).

\section{References}

[1] Williamson EJ, Walker AJ, Bhaskaran K, et al. Factors associated with COVID-19-related death using OpenSAFELY. Nature. 2020;584(7821):430-436.

[2] Richardson $S$, Hirsch JS, Narasimhan $M$, et al. Presenting characteristics, comorbidities, and outcomes among 5700 patients hospitalized with COVID19 in the New York city area. JAMA. 2020;323(20): 2052-2059.

[3] Wu C, Chen X, Cai Y, et al. Risk factors associated with acute respiratory distress syndrome and death in patients with coronavirus disease 2019 pneumonia in Wuhan, China. JAMA Intern Med. 2020;180(7): 934-943.

[4] Batty GD, Deary IJ, Luciano M, et al. Psychosocial factors and hospitalisations for COVID-19: prospective cohort study based on a community sample. Brain Behav Immun. 2020;89:569-578.

[5] Taquet M, Luciano S, Geddes JR, et al. Bidirectional associations between COVID-19 and psychiatric disorder: retrospective cohort studies of 62354 COVID19 cases in the USA. Lancet Psychiatry. 2020;8: 130-140.

[6] Wang Q, Xu R, Volkow ND. Increased risk of COVID-19 infection and mortality in people with mental 
disorders: analysis from electronic health records in the United States. World Psychiatry. 2021;20(1): 124-130.

[7] Batty GD, Gale CR. Pre-pandemic mental illness and risk of death from COVID-19. Lancet Psychiatry. 2021; 8(3):182-183.

[8] Mazereel V, Van Assche K, Detraux J, et al. COVID-19 vaccination for people with severe mental illness: why, what, and how? Lancet Psychiatry. 2021;8: 444-450.

[9] Osborn DP, Nazareth I, King MB. Physical activity, dietary habits and coronary heart disease risk factor knowledge amongst people with severe mental illness: a cross sectional comparative study in primary care. Soc Psychiatry Psychiatr Epidemiol. 2007;42(10): 787-793.

[10] Bradshaw T, Mairs H. Obesity and serious mental ill health: a critical review of the literature. Healthcare. 2014;2(2):166-182.

[11] Russ TC, Stamatakis E, Hamer $M$, et al. Association between psychological distress and mortality: individual participant pooled analysis of 10 prospective cohort studies. Bmj. 2012;345(jul31 4):e4933-e4933.

[12] Howard LM, Barley EA, Davies E, et al. Cancer diagnosis in people with severe mental illness: practical and ethical issues. Lancet Oncol. 2010;11(8):797-804.

[13] Parker MA, Robinson $M H$, Scholefield JH, et al. Psychiatric morbidity and screening for colorectal cancer. J Med Screen. 2002;9(1):7-10.

[14] Lorenz RA, Norris MM, Norton LC, et al. Factors associated with influenza vaccination decisions among patients with mental illness. Int J Psychiatry Med. 2013;46(1):1-13.

[15] Murphy J, Vallières F, Bentall RP, et al. Psychological characteristics associated with COVID-19 vaccine hesitancy and resistance in Ireland and the United Kingdom. Nat Commun. 2021;12(1):29.

[16] Jefsen $\mathrm{OH}$, Kølbaek P, Gil Y, et al. COVID-19 vaccine willingness amongst patients with mental illness compared with the general population. Acta Neuropsychiatr. 2021;33(5):273-276.

[17] Nguyen KH, Srivastav A, Razzaghi H, et al. COVID-19 vaccination intent, perceptions, and reasons for not vaccinating among groups prioritized for early vaccination - United States, September and December 2020. MMWR Morb Mortal Wkly Rep. 2021;70(6): 217-222.

[18] Ruiz JB, Bell RA. Predictors of intention to vaccinate against COVID-19: results of a nationwide survey. Vaccine. 2021;39(7):1080-1086.

[19] Lynn P. Sample design for Understanding Society. Understanding Society Working Paper Series No. 2009 - 01. Institute for Social and Economic Research, University of Essex; 2009

[20] How Understanding Society: the UK Household Longitudinal Study adapted to the COVID-19 pandemic. Survey Research Methods. 2020; 14;235-239.\}

[21] Robertson E, Reeve KS, Niedzwiedz CL, et al. Predictors of COVID-19 vaccine hesitancy in the UK household longitudinal study. Brain Behavior Immunity. 2021;94:41-50.
[22] Gallacher J. Covid-19: Oxford University vaccine is highly effective (BBC News). 2020. https:// wwwbbccouk/news/health-55040635

[23] Stuart AL, Pasco JA, Jacka FN, et al. Comparison of selfreport and structured clinical interview in the identification of depression. Compr Psychiatry. 2014;55(4):866-869.

[24] Holi MM, Marttunen M, Aalberg V. Comparison of the GHQ-36, the GHQ-12 and the SCL-90 as psychiatric screening instruments in the Finnish population. Nord J Psychiatry. 2003;57(3):233-238.

[25] Hankins $M$. The factor structure of the twelve item general health questionnaire (GHQ-12): the result of negative phrasing? Clin Pract Epidemiol Ment Health. 2008;4(1):10-18.

[26] Russ T, Hamer M, Stamatakis E, et al. Psychological distress as a risk factor for dementia death. Arch Intern Med. 2011;171(20):1858-1859.

[27] Russ TC, Kivimaki M, Morling JR, et al. Association between psychological distress and liver disease mortality: a meta-analysis of individual study participants. Gastroenterology. 2015;148(5):958-966.

[28] Kriegsman DM, Penninx BW, van Eijk JT, et al. Selfreports and general practitioner information on the presence of chronic diseases in community dwelling elderly. A study on the accuracy of patients' selfreports and on determinants of inaccuracy. J Clin Epidemiol. 1996;49(12):1407-1417.

[29] Smith GD, Spiegelhalter D. Shielding from covid-19 should be stratified by risk. BMJ. 2020;369:m2063.

[30] Batty GD, Deary IJ, Fawns-Ritchie C, et al. Pre-pandemic cognitive function and COVID-19 vaccine hesitancy: prospective cohort study. Brain Behav Immun 2021;96:100-105.

[31] Steptoe A, Breeze E, Banks J, et al. Cohort profile: the English longitudinal study of ageing. Int J Epidemiol. 2013;42(6):1640-1648.

[32] Borsch-Supan A, Brandt M, Hunkler C, et al. Data resource profile: the survey of health, ageing and retirement in Europe (SHARE). Int J Epidemiol. 2013; 42(4):992-1001.

[33] Richards M, Shipley B, Fuhrer R, et al. Cognitive ability in childhood and cognitive decline in mid-life: longitudinal birth cohort study. Bmj. 2004;328(7439):552.

[34] Sonnega A, Faul JD, Ofstedal MB, et al. Cohort profile: the health and retirement study (HRS). Int J Epidemiol. 2014;43(2):576-585.

[35] Lachman ME, Agrigoroaei S, Murphy C, et al. Frequent cognitive activity compensates for education differences in episodic memory. Am J Geriatr Psychiatry. 2010;18(1):4-10.

[36] Batty GD, Deary I, Gale C. Pre-pandemic cognitive function and COVID-19 mortality: prospective cohort study. medRxiv. 2021.

[37] Detoc M, Bruel S, Frappe $P$, et al. Intention to participate in a COVID-19 vaccine clinical trial and to get vaccinated against COVID-19 in France during the pandemic. Vaccine. 2020;38(45):7002-7006.

[38] Wang J, Jing R, Lai X, et al. Acceptance of COVID-19 vaccination during the COVID-19 pandemic in China Vaccines. 2020;8(3):482.

[39] Freeman D, Loe BS, Chadwick A, et al. COVID-19 vaccine hesitancy in the UK: the Oxford coronavirus 
explanations, attitudes, and narratives survey (oceans) II. Psychol Med. 2020;11:1-15.

[40] Williams L, Flowers P, McLeod J, et al. Social patterning and stability of intention to accept a COVID-19 vaccine in Scotland: will those most at risk accept a vaccine? Vaccines. 2021;9(1):17.

[41] Maguire PA, Reay RE, Looi JC. Nothing to sneeze at uptake of protective measures against an influenza pandemic by people with schizophrenia: willingness and perceived barriers. Australas Psychiatry. 2019; 27(2):171-178.

[42] Miles LW, Williams N, Luthy KE, et al. Adult vaccination rates in the mentally ill population: an outpatient improvement project. J Am Psychiatr Nurses Assoc. 2020;26(2):172-180.

[43] Gilbert RM, Mersky JP, Lee CP. Prevalence and correlates of vaccine attitudes and behaviors in a cohort of low-income mothers. Prev Med Rep. 2021;21:101292.
[44] Liao Q, Cowling BJ, Lam WW, et al. Factors affecting intention to receive and self-reported receipt of 2009 pandemic ( $\mathrm{H} 1 \mathrm{~N} 1)$ vaccine in Hong Kong: a longitudinal study. PLOS One. 2011;6(3):e17713.

[45] Harris KM, Maurer J, Lurie N. Do people who intend to get a flu shot actually get one? J Gen Intern Med. 2009;24(12):1311-1313.

[46] Fry A, Littlejohns TJ, Sudlow C, et al. Comparison of sociodemographic and Health-Related characteristics of UK biobank participants with those of the general Population. Am J Epidemiol. 2017;186(9):1026-1034.

[47] Batty GD, Gale CR, Kivimaki $M$, et al. Comparison of risk factor associations in UK biobank against representative, general population based studies with conventional response rates: prospective cohort study and individual participant meta-analysis. BMJ. 2020; 368:m131.

\section{Appendix. Batty GD, Deary IJ, Altschul D. Pre-pandemic mental and physical health as predictors of COVID-19 vaccine hesitancy: evidence from a UK cohort study}

Table a1. Odds ratios (95\% confidence intervals) for the relation of mental and physical health with later COVID-19 vaccine hesitancy in Understanding Society - with models featuring individual covariates $(N=7361)$.

\begin{tabular}{|c|c|c|c|c|c|c|c|}
\hline & $\begin{array}{c}\text { Number } \\
\text { hesitant/Total } \\
\text { at risk }\end{array}$ & $\begin{array}{l}\text { Age, sex, \& } \\
\text { ethnicity }\end{array}$ & $\begin{array}{l}\text { Age, sex, } \\
\text { ethnicity, \& } \\
\text { comorbidity }\end{array}$ & $\begin{array}{l}\text { Age, sex, } \\
\text { ethnicity, \& } \\
\text { shielding }\end{array}$ & $\begin{array}{l}\text { Age, sex, } \\
\text { ethnicity, \& } \\
\text { education }\end{array}$ & $\begin{array}{l}\text { Age, sex, } \\
\text { ethnicity, and } \\
\text { cognition }\end{array}$ & $\begin{array}{c}\text { All } \\
\text { covariates }\end{array}$ \\
\hline \multicolumn{8}{|l|}{ Psychiatric morbidity } \\
\hline Anxiety & $50 / 324$ & $1.00(0.72,1.36)$ & $1.06(0.76,1.44)$ & $1.00(0.72,1.36)$ & $1.04(0.74,1.42)$ & $1.02(0.73,1.39)$ & $1.11(0.79,1.52)$ \\
\hline Depression & $54 / 368$ & $0.99(0.72,1.33)$ & $1.05(0.77,1.43)$ & $0.99(0.72,1.34)$ & $1.02(0.74,1.38)$ & $1.03(0.75,1.39)$ & $1.12(0.81,1.53)$ \\
\hline $\begin{array}{l}\text { Other mental health } \\
\text { condition(s) }\end{array}$ & $20 / 111$ & $1.08(0.64,1.75)$ & $1.17(0.69,1.89)$ & $1.08(0.64,1.75)$ & $1.06(0.62,1.73)$ & $1.15(0.68,1.87)$ & $1.21(0.71,1.97)$ \\
\hline $\begin{array}{l}\text { Any mental health } \\
\text { condition }\end{array}$ & $71 / 491$ & $0.99(0.75,1.29)$ & $1.05(0.79,1.37)$ & $0.99(0.75,1.29)$ & $1.04(0.78,1.35)$ & $1.05(0.79,1.37)$ & $1.14(0.86,1.49)$ \\
\hline \multicolumn{8}{|l|}{ Psychological distress } \\
\hline Asymptomatic (score 0) & $443 / 3339$ & 1.0 (ref) & 1.0 & 1.0 & 1.0 & 1.0 & 1.0 \\
\hline $\begin{array}{l}\text { Subclinically } \\
\text { symptomatic (1-3) }\end{array}$ & $247 / 2256$ & $0.77(0.64,0.91)$ & $0.77(0.68,0.96)$ & $0.77(0.60,0.94)$ & $0.79(0.62,0.97)$ & $0.79(0.62,0.96)$ & $0.81(0.63,0.98)$ \\
\hline Symptomatic (4-6) & $90 / 750$ & $0.77(0.59,0.98)$ & $0.78(0.63,1.05)$ & $0.77(0.52,1.02)$ & $0.78(0.53,1.04)$ & $0.78(0.53,1.04)$ & $0.82(0.56,1.07)$ \\
\hline Highly symptomatic (7-12) & $173 / 1016$ & $1.05(0.85,1.28)$ & $1.08(0.91,1.38)$ & $1.06(0.86,1.26)$ & $1.07(0.87,1.27)$ & $1.07(0.86,1.27)$ & $1.12(0.92,1.33)$ \\
\hline$P$ for quadratic & & $<0.0001$ & 0.017 & 0.028 & 0.034 & 0.036 & 0.003 \\
\hline$P$ for linear trend & & 0.251 & 0.099 & 0.148 & 0.147 & 0.145 & 0.075 \\
\hline $\begin{array}{l}\text { Per SD (3.5 points) } \\
\text { decrease }\end{array}$ & $953 / 7361$ & $0.93(0.81,1.06)$ & $0.91(0.79,1.04)$ & $0.92(0.80,1.05)$ & $0.92(0.80,1.05)$ & $0.92(0.80,1.05)$ & $0.88(0.75,1.02)$ \\
\hline \multicolumn{8}{|l|}{ Physical morbidity } \\
\hline Cardiometabolic disease & $147 / 1905$ & $0.82(0.67,1.00)$ & $0.82(0.67,1.00)$ & $0.83(0.68,1.01)$ & $0.80(0.66,0.98)$ & $0.78(0.64,0.95)$ & $0.78(0.64,0.95)$ \\
\hline Respiratory disease & $107 / 1034$ & $0.71(0.57,0.88)$ & $0.71(0.57,0.88)$ & $0.72(0.58,0.90)$ & $0.73(0.58,0.90)$ & $0.72(0.57,0.89)$ & $0.74(0.59,0.93)$ \\
\hline Any cancer & 29/389 & $0.87(0.58,1.28)$ & $0.87(0.58,1.28)$ & $0.89(0.59,1.30)$ & $0.90(0.59,1.32)$ & $0.92(0.61,1.34)$ & $0.95(0.62,1.39)$ \\
\hline $\begin{array}{l}\text { Any physical health } \\
\text { condition }\end{array}$ & $225 / 2389$ & $0.72(0.61,0.85)$ & $0.72(0.61,0.85)$ & $0.73(0.61,0.86)$ & $0.72(0.61,0.85)$ & $0.71(0.60,0.83)$ & $0.72(0.60,0.85)$ \\
\hline Shielding in household & $196 / 1383$ & $0.81(0.63,1.03)$ & $0.81(0.63,1.03)$ & & $0.78(0.61,1.00)$ & $0.76(0.59,0.97)$ & $0.76(0.59,0.96)$ \\
\hline
\end{tabular}

All covariates are: age, sex, ethnicity, education, shielding, and cognitive function. Effect estimates for physical morbidity and psychiatric morbidity were mutually-adjusted. 